\title{
Role of Bioreactors in Regeneration of Articular Cartilage
}

\author{
Farhana Yasmin $^{1 *}$ and Xiongbiao Chen ${ }^{1,2}$ \\ ${ }^{1}$ Division of Biomedical Engineering, College of Engineering, University of Saskatchewan, \\ 1A26, 57 Campus Drive, Saskatoon, SK, S7N 5A9, Canada \\ ${ }^{2}$ Department of Mechanical Engineering, College of Engineering, University of Saskatchewan, \\ 3B47, 57 Campus Drive, Saskatoon, SK, S7N 5A9, Canada
}

(Received February 1, 2016; accepted July 1, 2016)

Keywords: bioreactor, cartilage, chondrogenesis, sensing technology, mechanical stimulation

Interest in cartilage defect repair has been rapidly increasing with the growing number of sportsrelated injuries, traumas, congenital defects, and pathological disorders, for example, resulting in osteoarthritis (OA), a complex degenerative joint disease that affects a large number of people every year. Although modern medicine has entered into an advanced stage, current surgical procedures still have several limitations. At present, among all therapeutic approaches, tissue engineering has shown great potential for cartilage joint repair. Several methodologies of utilizing cells, scaffolds, and signalling molecules have been explored and developed. However, in regenerative cartilage therapies, one of the major challenges yet to be overcome is its inability to regenerate functional tissue owing to poor mass transfer, and limited ability in controlling physiocochemical cultural parameters during in vitro cell culture. To solve these problems, bioreactors can play a promising role because of their ability to control physiocochemical culture parameters and to provide mechanical stimulation, thereby inducing improved chondrogenesis of tissue-engineered cartilage. In this paper, we review the role of bioreactors in repairing articular cartilage defects with recent advancements in these two areas.

\section{Introduction}

Cartilage defect repair is challenging because of its avascular nature, poor regenerative capacity, complex stratified architecture, disparate biomechanical property, ${ }^{(1)}$ and intrinsic mesenchymal stem cell population. ${ }^{(2)}$ Physical trauma to the knee or other articular joints and degenerative joint diseases can lead to several types of focal cartilage defects affecting both articular cartilage and the underlying subchondral bone, thereby causing osteoarthritis (OA). At present, more than 250000 knee and hip replacement surgeries are performed in the United States each year, and it is expected that with the increase in the aging population and obesity, the number of osteoarthritis cases will increase drastically in the future. ${ }^{(3)}$ It has been anticipated that by 2030, an estimated 65 million adult Americans will be diagnosed with osteoarthritis. ${ }^{(4)}$ As a result, osteochondral defects resulting from trauma, osteoarthritis, congenital defects, and/or pathological disorders are areas of increasing clinical interest. The existing therapies for cartilage defect repair (e.g., chondral shaving, abrasion arthroplasty, subchondral drilling, microfracturing, mosaicplasty, prosthetic joint replacement, subchondral abrasion, pridie perforations transplantation of osteochondral plugs, and autologous

"Corresponding author: e-mail: fay610@mail.usask.ca 
chondrocyte implantation) are still challenging because of their various limitations, such as poor donor tissue availability, donor site morbidity, disease transmission, implant loss, limited durability of prosthetics, and requirement to undergo many surgeries. . $^{(1,5)}$

At present, among all therapeutic approaches, tissue engineering has shown great potential for cartilage joint repair. In cartilage tissue engineering, articular cartilage can be regenerated by two possible approaches. Among them, the first approach has been termed autologous chondrocyte implantation (ACI), where expanded articular chondrocytes are implanted under a periosteal flap after surgical excision of the lesion. The second approach aims to regenerate neocartilaginous tissue by combining three basic components of tissue engineering: a suitable biocompatible scaffold; cells and an environment including mechanical stimuli; and a combination of appropriate bioactive molecules, e.g., growth factor and proteins (see Fig. 1). It has been expected that, through dynamic interaction of these components, an artificial transplantable tissue construct can be regenerated that might show enhanced integration with native articular cartilage. ${ }^{(2,6-8)}$

Both types of cell-based approaches used for regenerating articular cartilage involve the isolation of cells and subsequent expansion in vitro. Therefore, in tissue engineering of articular cartilage, in addition to requiring adequate biofunctional proliferable cell sources and bioactive signalling molecules, an appropriate culture environment is also important for generating 3D cartilage constructs in vitro. Moreover, the development of engineered construct largely depends on the hydrodynamic environment and adequate supply of nutrients, including oxygen and glucose, to the cells during cell culture in vitro. ${ }^{(9,10)}$ However, owing to the avascular nature of tissue-engineered cartilage, the supply of nutrients and the metabolic waste removal depend on diffusion. This creates gradients of nutrients and waste throughout the entire engineered constructs. If tissue-engineered cartilage constructs are incubated under a static culture condition, significant diffusion resistance is induced. This resistance exists through the static medium and cannot be overcome, resulting in poor nutrient and waste transport to and from the engineered cartilage. ${ }^{11,12)}$ Also, high initial cell density is essential for the fabrication of articular cartilage during cell culture. However, during

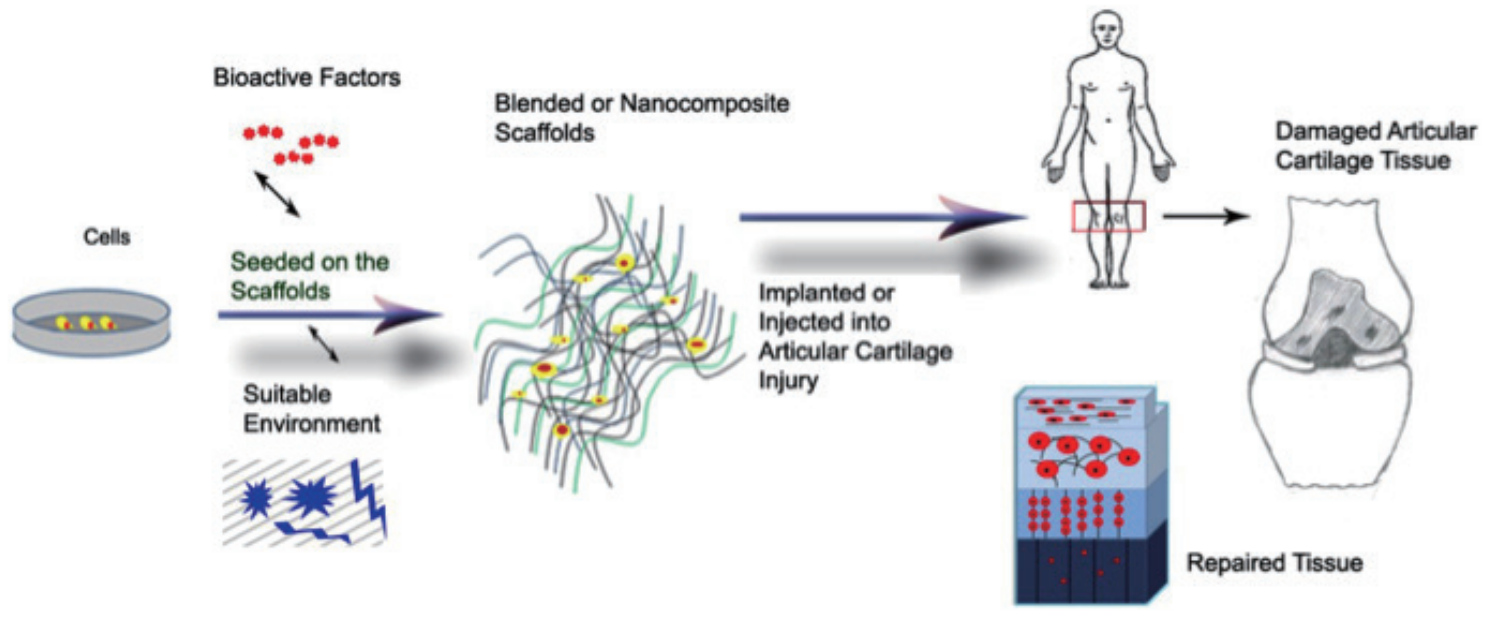

Fig. 1. (Color online) Schematic representation of the articular-tissue-engineering procedure using three key components of tissue engineering (cells, bioactive factors, and mechanical stimuli). ${ }^{(7)}$ 
monolayer culture under the static condition, chondrogenesis also decreases with increasing cell density owing to the poor permeability of the culture medium through the constructs, resulting in a limited supply of nutrients to cells. As a result, cartilage synthesis in vitro has been one of the major limitations for obtaining high-quality engineered cartilage constructs. ${ }^{(13,14)}$

To address these limitations, one approach may be to utilize bioreactors to provide environmental conditions that stimulate those of chondrogenesis. ${ }^{(15)}$ Through the creation of an in vitro cell culture process inside a bioreactor under dynamic flow conditions, it is possible to facilitate mass transfer and control both microenvironmental parameters, such as temperature, $\mathrm{pH}$, pressure, oxygen tension, metabolites, regulatory molecules, shear stress, and electrical pacing, and aseptic parameters, such as feeding, waste removal, and sampling resulting in increased production of cartilage specific matrix, proteins. ${ }^{(3,10,14)}$ There are several types of bioreactors used in tissue engineering. However, only bioreactors that have been used for cartilage tissue engineering will be discussed in this review. ${ }^{(14,16)}$ Therefore, the main body of this literature review will focus on the important features of bioreactor design and the role of bioreactors in the improvement of cartilaginous extracellular matrix, with a detailed overview of recent and promising advancements in this area. Finally, the review will be concluded with future recommendations in this particular area.

\section{Important Features for Bioreactor Design}

In tissue engineering, bioreactors are used for different purposes, including cell proliferation, generation of 3D tissue constructs, and as a direct organ support device. One of the critical issues that bioreactors face involves limited mass transfer, such as the supply of oxygen and nutrient, and the removal of toxic metabolites, resulting in poor tissue-engineered constructs. Furthermore, each type of tissue construct requires a specific bioreactor design. ${ }^{(17)}$ Therefore, tissue-specific bioreactors should be designed on the basis of a comprehensive understanding of the biological and engineering aspects. Figure 2 shows a schematic representation of a bioreactor. The construction idea behind the design of a bioreactor should be such that it mimics the native environment of articular cartilage, such as oxygen tension, $\mathrm{pH}$, temperature, and shear stress..$^{(9)}$ Generally, the culture environment of a bioreactor consists of a temperature of $37{ }^{\circ} \mathrm{C}, 90$ to $100 \%$ relative humidity, and a gas-phase mixture comprising of $20 \% \mathrm{O}_{2}$ and $5 \% \mathrm{CO}_{2} \cdot{ }^{(15)}$ For improved mass transfer of small and large molecules into the cartilage matrix, bioreactors should be able to apply hydrostatic force through mechanical stimulation. As a homogeneous distribution of cells within the scaffold is essential, it should have the ability to distribute cells in a homogeneous fashion to enhance cartilage-specific matrix production.

Another essential feature that bioreactors should have is the capability to seed scaffolds in an automated and controlled manner. Under such a condition, nutrient and waste transfers are automatically regulated by the flow of the growth medium. To this end, sensing technologies integrated in bioreactors can play a significant role in the automation and in-process control of tissue manufacturing processes. Using sensors, it is possible to monitor and control the basic parameters of culture medium, such as temperature, $\mathrm{pH}$, pressure, oxygen tension, metabolites, regulatory molecules, shear stress, and electrical pacing. Sensors used in bioreactors can be divided into three types: (i) invasive sensors, (ii) noninvasive sensors, and (iii) shunt sensors. Whereas invasive sensors are placed directly inside the culture chamber of the bioreactor, noninvasive 


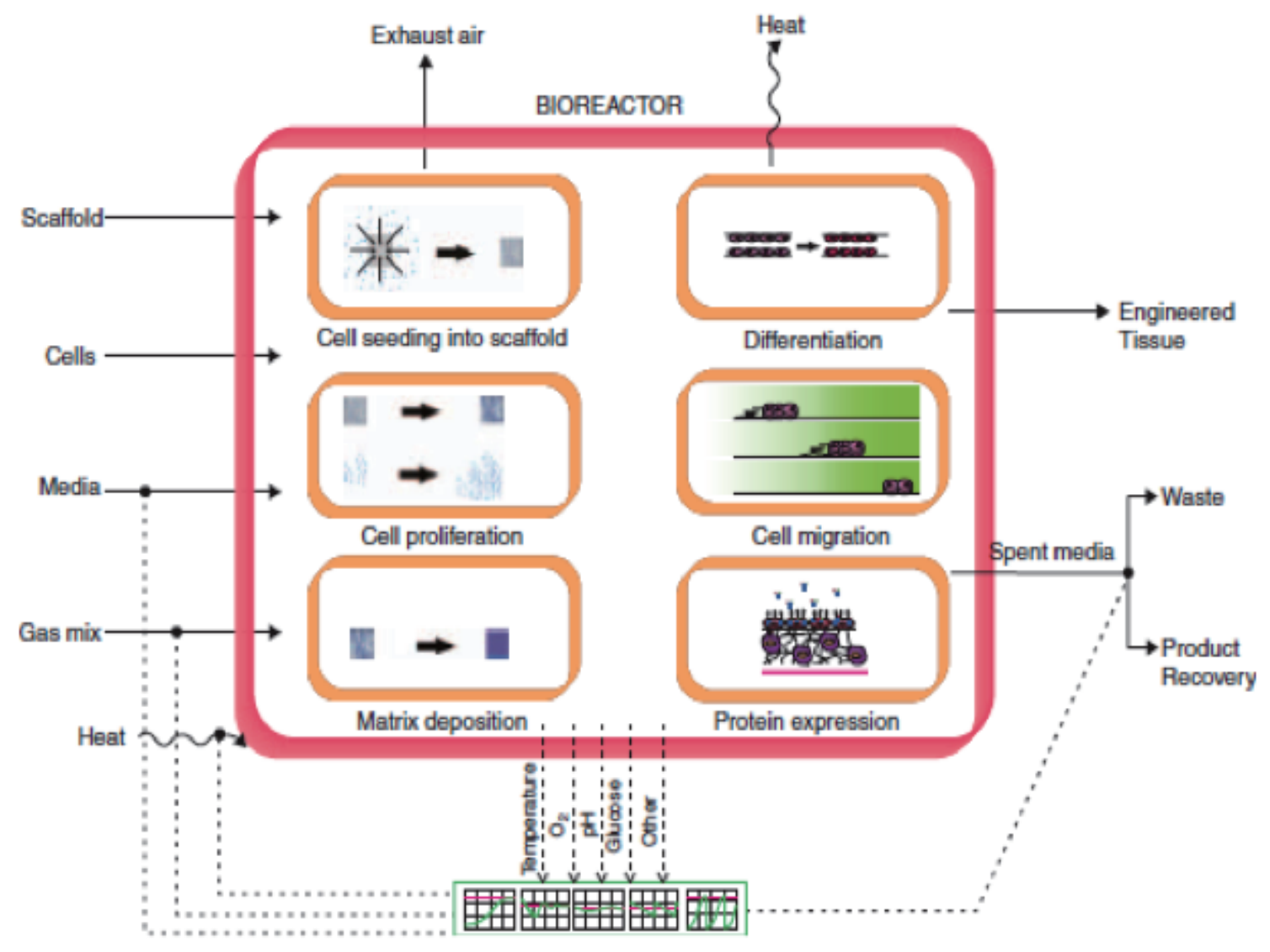

Fig. 2. (Color online) Schematic representation of a bioreactor. ${ }^{(18)}$

(noncontact) sensors do not require close contact with the interior of the culture chamber. In shunt sensors, the analysis of culture medium is performed either manually or automatically, online, with the help of common instruments used in bio-analytics. ${ }^{(16,19)}$

The features described above are important because they not only limit the risk of contamination but also ensure controlled, reproducible cost-effective tissue-engineered constructs. ${ }^{(9,15)}$ A future bioreactor system should be such that it is integrated into the entire cultivation scheme comprising biopsy, proliferation, cell seeding, tissue formation, and delivery to the site of application (Fig. 3).

\section{Bioreactors Used in Cartilage Tissue Engineering}

Bioreactors used for cartilage tissue engineering include the parallel-plate bioreactor, rotatingwall vessel (RWV) bioreactor, concentric-cylinder bioreactor, wavy-walled bioreactor, perfusionbased bioreactor, compression bioreactor, double-chambered bioreactor, and hydrostatic pressure bioreactor (see Fig. 4). ${ }^{(20,21)}$ There are also some specially designed bioreactors that can be used to apply controlled mechanical simulation to engineered cartilage constructs. These will be discussed in Sect. 4. 


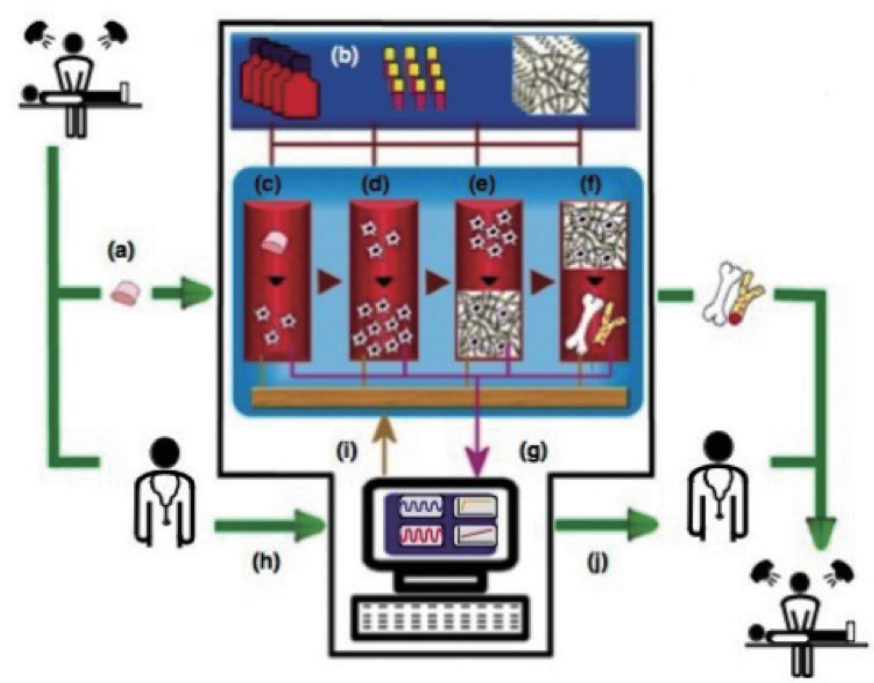

Fig. 3. (Color online) Closed-system bioreactor integrated into the total cultivation scheme comprising biopsy, proliferation, cell seeding, tissue formation, and delivery to the site of application. ${ }^{(11)}$

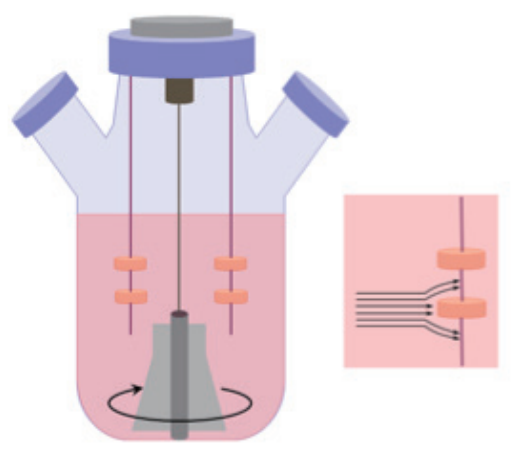

(a)

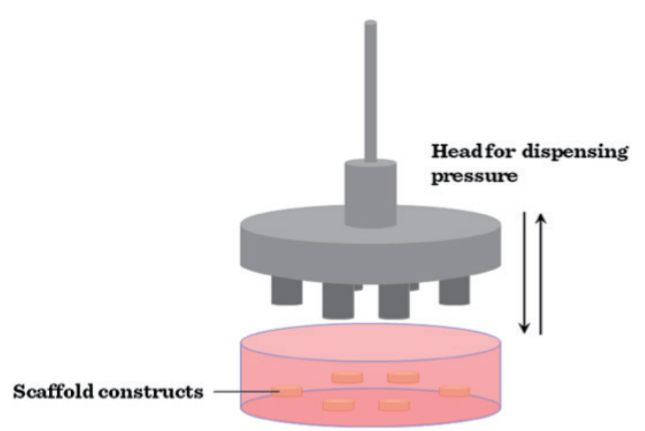

(c)

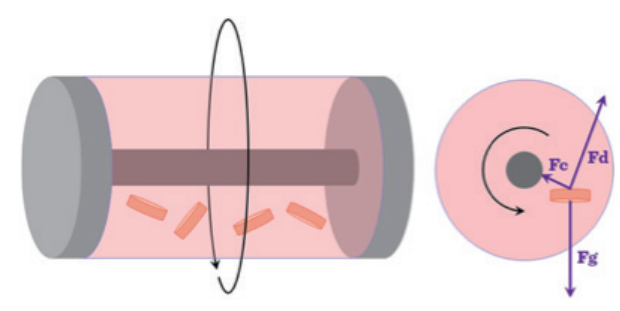

(b)

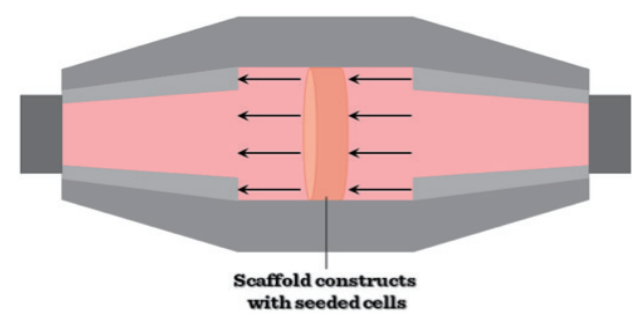

(d)

Fig. 4. (Color online) Bioreactors used for cartilage tissue engineering. (a) Spinner flask bioreactor. (b) RWV bioreactor. (c) Compression bioreactor. (d) Flow perfusion bioreactor. ${ }^{(21)}$ 


\subsection{Spinner flask bioreactor}

Spinner flask bioreactors [Fig. 4(a)] are the simplest and the most frequently used bioreactor types. In these bioreactors, scaffolds are often suspended and a magnetic stirrer mixes the medium across the scaffolds. By using this type of bioreactor, it is possible to mix oxygen and nutrients homogeneously and reduce the concentration boundary layer at the construct surface, resulting in improved chondrocyte proliferation and extracellular matrix (ECM) deposition. ${ }^{(21,22)}$ Chang et al. produced engineered cartilage by culturing under either the static or dynamic condition, using a spinner flask bioreactor. In comparison to static culture, chondrocytes cultured in the spinner flask bioreactors were uniformly distributed in the scaffold, resulting in improved chondrogenesis. ${ }^{(23)}$

Shear stress plays an important role in cartilage tissue engineering. Whereas low shear stress stimulates ECM synthesis and deposition, high shear stress suppresses glycosamino-glycan (GAG) deposition. One of the drawbacks of this bioreactor is that it applies high shear stress, resulting in decreased GAG deposition. ${ }^{(21)}$ For example, a group of researchers cultured chondrocytes in spinner flasks either statically or at different turbulent mixing intensities and observed that, in comparison to the case of the static condition, constructs cultured with different mixing intensities resulted in higher amounts of collagen. GAG was released, but only a small fraction of GAG was retained in the construct. This may be due to the high shear stress, which suppresses GAG deposition, applied by this bioreactor. ${ }^{(3)}$ Another drawback of this bioreactor is that its cell seeding efficiency is typically low, resulting in inhomogeneous cell distribution throughout the scaffolds, which leads to a poor tissue-engineered construct. Therefore, at present, the use of this bioreactor in cartilage tissue engineering is limited. ${ }^{(21)}$

\subsection{RWV bioreactor}

Another type of novel bioreactor that is used in cartilage tissue engineering is the RWV bioreactor [see Fig. 4(b)]. In this type of bioreactors, scaffolds are free to move and remain in a state of free fall. In comparison to turbulent flow produced within stirred flasks, the dynamic laminar flow produced with this bioreactor supports reduced levels of shear stress, and reduces the diffusional limitations of nutrient and waste transfer, thereby ensuring uniform cell distribution and the formation of cartilaginous tissues containing higher amounts of more uniformly distributed GAG and collagen. ${ }^{(8,9,11,24)}$ Therefore, the use of this bioreactor enables the fabrication of cartilaginous constructs with improved biochemical and biomechanical properties. ${ }^{(11)}$ Tognana et al. used the RWV bioreactor to culture chondrocytes, resulting in the formation of cartilaginous tissues containing higher amounts of more uniformly distributed GAG. ${ }^{(25)}$ To show the effect of this bioreactor on chondrogenesis, another research group cultured chondrocytes both under the static condition and using the RWV bioreactor. While condrocyte dedifferentiation occurred in static media, upon transferring these dedifferentiated cells to the RWV bioreactor, the cells showed increased aggregation and type II collagen expression. ${ }^{(26)}$

\subsection{Concentric-cylinder bioreactor}

Concentric-cylinder bioreactors have been developed to culture tissue-engineered constructs of articular cartilage under a hydrodynamic environment. This bioreactor operates under low shear 
stress. Shear stress can be controlled by varying the rotation speed, allowing dynamic cell seeding and uniform loading of constructs. ${ }^{(27,28)}$ Saini and Wick used a concentric-cylinder bioreactor to seed bovine chondrocytes into porous poly-lactic acid constructs. Using this bioreactor, it was possible to seed cells with an efficiency greater than $95 \%$, thereby stimulating chondrocytic proliferation and matrix deposition. ${ }^{(29)}$ The native environment of cartilage is low in oxygen and by using this bioreactor, it is possible to maintain low oxygen tension (5\%), leading to greater proteoglycans synthesis. To show the effect of oxygen tension on chondrogenesis using this concentric-cylinder bioreactor, the same group of researchers cultured scaffolds operated at 5 or $20 \%$ oxygen and observed that, in comparison to constructs using the bioreactor operated with $20 \%$ oxygen tension, the construct obtained with the bioreactor operated with $50 \%$ oxygen tension had a $65 \%$ higher deposition rate of GAG. ${ }^{(29)}$

\subsection{Wavy-walled bioreactor}

Another type of bioreactor used in the cultivation of cartilage constructs is the wavy-walled bioreactor. With this bioreactor, by applying reduced shear stress and high-axial mixing, it is possible to increase cell proliferation and dedifferentiation, resulting in increased production of the ECM of cartilage protein. ${ }^{30,31)}$ Compared with the spinner flask reactor, wavy-walled bioreactor has a significant effect on chondrogenesis because of its increased ECM deposition. Bueno et al. cultured constructs containing chondrocytes in both the spinner flask reactor and the wavy-walled bioreactor. After 4 weeks of culture, it was observed that, in comparison to the construct obtained with the spinner flask reactor, the construct cultured in the wavy-walled bioreactor was thicker, owing to the increased cell proliferation and differentiation capacity. ${ }^{(30)}$

\subsection{Compression bioreactor}

This type of bioreactor can be designed such that both static and dynamic load can be applied to a cartilage construct [Fig. 4(c)]. However, in contrast to static loading, dynamic loading is more beneficial for generating full-thickness articular cartilage because of its improved mass transfer. Using this bioreactor, load can be transferred evenly to the cell-seeded constructs via flat platens. Another advantage of this bioreactor is that it enables the culture of multiple scaffolds at the same time. It is also possible to provide mechanical stimulation in cells of scaffolds, resulting in improved chondrogenesis owing to increased cellular proliferation, differentiation, and type II collagen expression. ${ }^{(21)}$

\subsection{Double-chambered bioreactor}

Currently, another type of novel bioreactor system that has been developed for cartilagetissue engineering is the double-chambered bioreactor. A special feature of this bioreactor is that by maintaining an appropriate cultural condition, it is possible to culture different cell types simultaneously. ${ }^{(21)}$ To fabricate a biphasic osteochondral construct, Chang et al. designed a novel double-chambered bioreactor for simultaneous coculture of chondrocytes and osteoblasts. After 4 weeks of culture, hyaline-like cartilage was formed, suggesting a potential for osteochondral defect repair. $^{(32)}$ 


\subsection{Perfusion-based bioreactor}

In tissue engineering, among all types of bioreactors discussed above, perfusion-based bioreactors [Fig. 4(d)] have been extensively used because of their improved fluid transport capacity compared with other bioreactors. With this bioreactor, it is possible for the culture medium to flow through the pores of a solid scaffold to facilitate cell differentiation by increasing nutrient transport to the scaffold interior and by providing mechanical stimulation in the form of liquid, resulting in increased cell proliferation and biochemical content. ${ }^{(14,21,33)}$ A perfusion-based bioreactor can thus provide a well-controlled physicochemical dynamic culture environment, providing great potential to generate cartilage grafts of clinically relevant size. It has been reported that through the perfusion of chondrocyte-seeded scaffolds, it is possible to enhance cell survival, growth, and distribution of viable human chondrocytes homogeneously, resulting in elevated expression of type II collagen, increased sulfated GAG synthesis, and retention within the ECM with biomechanical properties close to those of native cartilage. ${ }^{(11,14,34-38)}$ To investigate the effect of perfusion on chondrogenesis, Davisson et al. seeded chondrocytes on a PGA scaffold and cultured the construct in a perfusionbased bioreactor. Although initially the synthesis and retention of GAG contents were decreased compared with the control, over time, GAG synthesis and deposition increased to approximately $40 \%$ within tissue-engineered constructs compared with static controls. ${ }^{(39)}$ Another research group also examined the efficacy of the perfusion-based bioreactor for chondrogenesis and obtained similar results. ${ }^{(40)}$ For the generation of articular cartilage, Carver and Heath designed a perfusionbased bioreactor that allows the application of intermittent hydrostatic pressure at physiological levels. To do this, the cell/scaffold constructs were subjected to an intermittent hydrostatic pressure of 500. The resulting construct increased the production of cartilaginous ECM. ${ }^{(41)}$ It was found that articular chondrocytes only produce type II collagen in a perfused environment. On the other hand, type II collagen was absent under the static culture condition. Forsey et al. encapsulated chondrocyte cells in alginate-chitosan and cultured the resulting construct under either the static or perfusion condition to show the expression of SOX-9 and type II collagen. Even though cells cultured in the perfusion-based bioreactor expressed both SOX-9 and type II collagen, cells cultured under the static condition expressed only SOX-9.(42) More recently, Gilbert et al. designed a fullthickness perfusion-based bioreactor (see Fig. 5) for the generation of a scaffold-free construct of articular cartilage under several conditions. In contrast to immediate perfusion after cell seeding in the construct, the best result was obtained when perfusion was delayed, resulting in a 24-mmdiameter scaffold-free tissue-engineered cartilage construct with improved biochemical and biomechanical properties. ${ }^{(43)}$

\section{Mechanical Stimulation Using Bioreactors}

Mechanical stimulation is a key determinant in the development and remodelling of tissueengineered blood vessels and cartilage. It plays a profound role in musculoskeletal tissue engineering, cartilage formation, and cardiovascular tissues. Various studies have shown that exposure to mechanical stimulation (e.g., mechanical compression, hydrodynamic pressure, and fluid flow), which are important modulators of cell physiology, can enhance the biochemical and mechanical properties of engineered cartilage. . $8,9,14,41,44)$ In native cartilage, in vivo physical stimulation mediates chondrocyte function by affecting mechanotransduction. Therefore, to improve chondrogenesis in vitro, bioreactors can be designed in such a way that they can transmit 


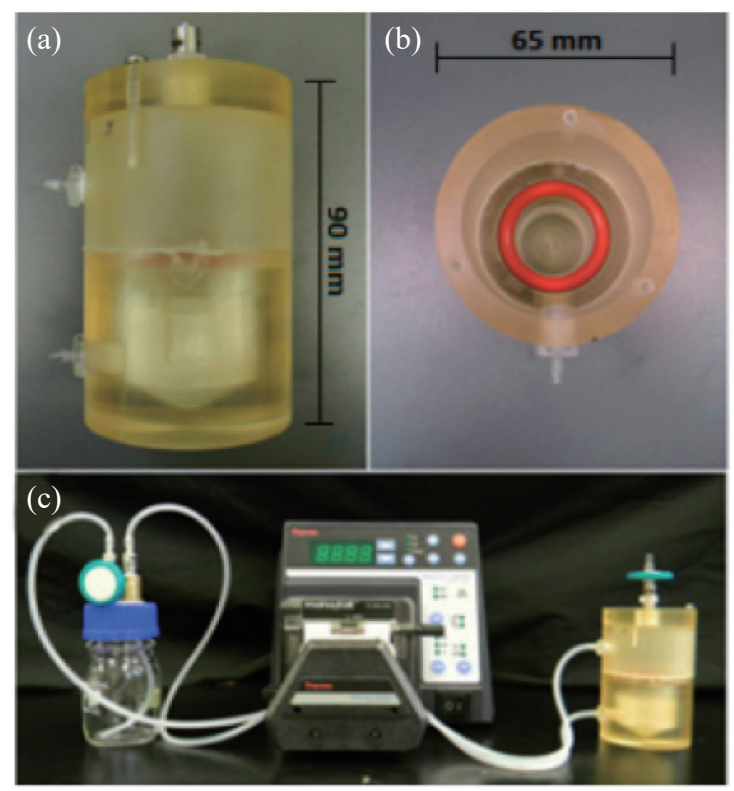

Fig. 5. (Color online) Design of through-thickness perfusion-based bioreactor. (a) Side view. (b) Top-down view. (c) Bioreactor setup. ${ }^{(43)}$

forces, such as shear, hydrostatic pressure, and compression forces and their combination to cartilage explants. Schulz and Bader provided a detailed overview on how various mechanical forces can be applied using a bioreactor. ${ }^{(15)}$ The mechanical stress that can be applied by various bioreactors can be classified on the basis of the principal type of stress being applied, as shown in Fig. $6{ }^{(15)}$

In cartilage tissue engineering, the most appropriate type of stimulation is dynamic compression loading because of its stimulating impact on tissue-engineered cartilage construct that enhances cartilage-specific ECM synthesis. ${ }^{(28)}$ Whereas static compression decreases biosynthetic activity, dynamic compression increases biosynthesis. Lee et al. examined the effect of static and dynamic loading on the cartilage construct and showed that dynamic compression loading increased protein and proteoglycan biosynthesis. On the other hand, static loading decreased the synthesis of protein and proteoglycan. ${ }^{(4)}$ To examine the effect of long-term confined dynamic compression on the engineered cartilage construct, Wernike et al. designed a bioreactor to apply dynamic compression onto the cell-seeded tissue-engineered construct. In addition to stabilizing the chondrocytic phenotype, the resulting construct showed increased GAG, type II collagen expression, and decreased type I collagen expression. ${ }^{(44)}$ Another research group also designed a novel bioreactor to provide hydro-dynamic compression to the tissue-engineered cartilage construct. The resulting construct showed increased cell proliferation with an increase in the compression modulus of the construct. However, one of the limitations of dynamic loading is its limited ability to retain GAG in the construct. ${ }^{(45)}$ To prevent reduced GAG deposition, hydrostatic-pressure bioreactors can be used because of their capacity to manipulate construct matrix accumulation. ${ }^{(27)}$ Using a bioreactor, $\mathrm{Hu}$ and Athanasiou applied intermittent compression of $10 \mathrm{MPa}$ and $1 \mathrm{~Hz}$ magnitude in cartilage constructs. In comparison to an unpressurized sample, samples that received intermittent 


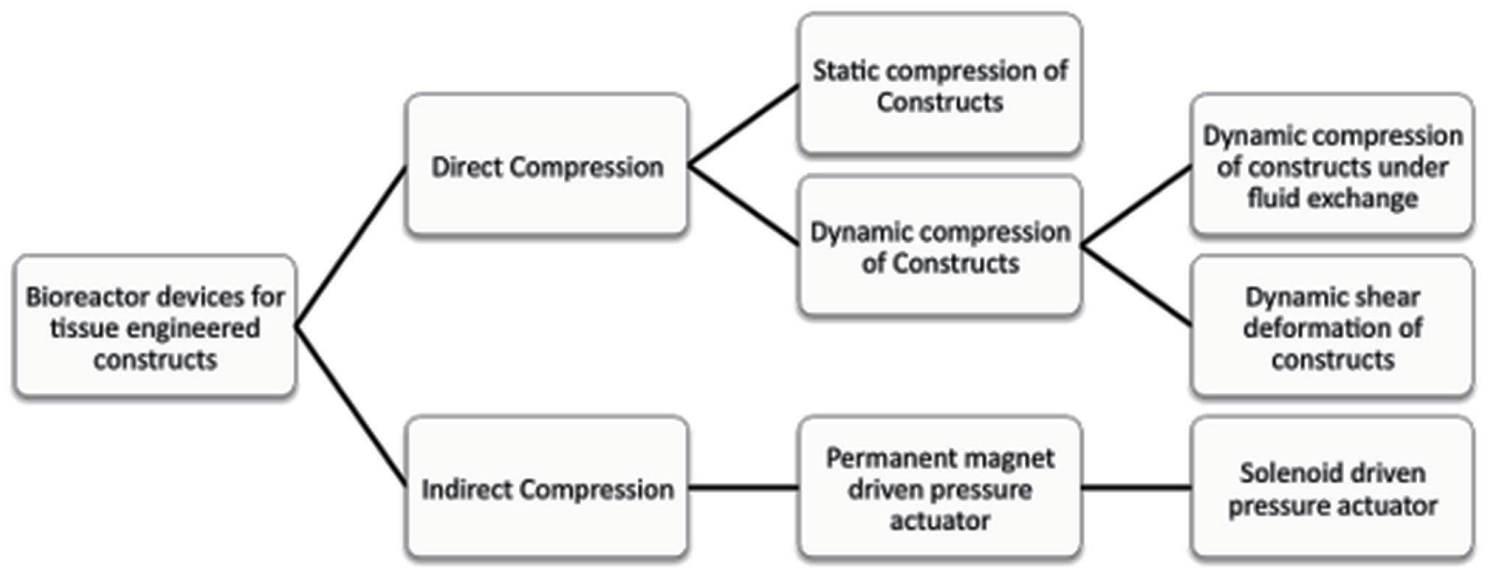

Fig. 6. Classification of various methods used in bioreactors for the compression of tissue engineered constructs on the basis of the principal type of stress being applied. ${ }^{(15)}$

hydrostatic pressure showed significant increases in collagen production and prevented the release of GAG from the construct. ${ }^{(17)}$ Another research group also performed a similar type of experiment under dynamic hydrodynamic loading conditions and obtained similar results with improved chondrogenesis. ${ }^{(27)}$

\section{Conclusions and Future Recommendations}

Tissue engineering plays a significant role in cartilage tissue engineering by combining its three key components: cells, scaffolds and signalling factors. However, in cartilage tissue engineering, one of the major limitations yet to be overcome is the inability to regenerate tissue with the original composition and structure of articular cartilage owing to poor cell viability and limited mass transfer during cell culture of large tissue-engineered constructs. To solve this problem, bioreactors can play a significant role because the basic parameters of the culture medium can be controlled, thereby improving cell viability by culturing cells in a dynamic environment. In addition to requiring adequate biofunctional proliferable cell sources and bioactive signalling molecules, an appropriate culture environment is also important for generating 3D cartilage constructs in vitro. Additionally, bioreactors can be used to generate three-dimensional functional tissues in vitro by increasing mass transfer through the constructs and providing mechanical stimulation. However, the goals and expectations of the development of bioreactors have only been partially fulfilled because of limited understanding of complex bioreactor design, and lack of knowledge about the effect of the microenvironment on cellular behavior within the bioreactors. When using bioreactors, it is important to determine the optimum engineering parameters, such as internal and external mass transfer, fluid velocity, shear stress, and electrical current distribution. It would be highly inefficient to determine the optimal engineering parameters by the traditional trial and error method, which would waste time and money. It is possible to predict the experimental results through mathematical modelling, thereby saving time and money. ${ }^{(9,14)}$ Moreover, by installing sensors in bioreactors it is possible to monitor and control the basic parameters in culture medium. However, the current 
sensing devices used in bioreactors are inadequate for obtaining real-time conditions within the bioreactor. To obtain the characteristics of the cellular product being manufactured within the bioreactors, new types of sensing devices, such as near-infrared and Raman spectroscopes can be integrated with bioreactors. ${ }^{(46)}$ Therefore, much work is required using both mathematical modelling and cutting-edge sensing devices to predict and control the optimum engineering parameters in order to improve the performance of bioreactors in cartilage tissue engineering.

\section{References}

1 Q. Yang, J. Peng, Q. Guo, J. Huang, L. Zhang, J. Yao, F. Yang, S. Wang, W. Xu, A. Wang, and S. Lu: Biomaterials 29 (2008) 2378.

2 Z. Izadifar, X. Chen, and W. Kulyk: J. Func. Biomater. 3 (2012) 799.

3 C. Chung and J. A. Burdick: Adv. Drug Deliv. Rev. 60 (2008) 243.

4 T. Holland, E. Bodde, V. Cuijpers, L. Baggett, Y. Tabata, A. Mikos, and J. Jansen: Osteo. Cart. 15 (2007) 187.

5 S.-J. Seo, C. Mahapatra, R. K. Singh, J. C. Knowles, and H.-W. Kim: J. Tissue Eng. 5 (2014) 1.

6 Z. Cao, C. Dou, and S. Dong: J. Nanomater. 2014 (2014).

7 A. H. Doulabi, K. Mequanint, and H. Mohammadi: Materials 7 (2014) 5327.

8 D. Nesic, R. Whiteside, M. Brittberg, D. Wendt, I. Martin, and P. Mainil-Varlet: Adv. Drug Deliv. Rev. 58 (2006) 300 .

9 R. Pörtner, S. Nagel-Heyer, C. Goepfert, P. Adamietz, and N. M. Meenen: J. Biosc. Bioeng. 100 (2005) 235.

10 M. S. Hossain, D. Bergstrom, and X. Chen: Biotech. Rep. 5 (2015) 55.

11 I. Martin, D. Wendt, and M. Heberer: Trends Biotech. 22 (2004) 80.

12 S. Zhou, Z. Cui, and J. P. Urban: Biotech. Bioeng. 101 (2008) 408.

13 L. Freed, J. Marquis, R. Langer, G. Vunjak-Novakovic, and J. Emmanual: Biotech. Bioeng. 43 (1994) 605.

14 N. Salehi-Nik, G. Amoabediny, B. Pouran, H. Tabesh, M. A. Shokrgozar, N. Haghighipour, N. Khatibi, F. Anisi, K. Mottaghy, and B. Zandieh-Doulabi: BioMed Res. Inter. 2013 (2013) 8946.

15 R. M. Schulz and A. Bader: Euro. Biophy. J. 36 (2007) 539.

16 D. Wendt, S. A. Riboldi, M. Cioffi, and I. Martin: Adv. Mater. 21 (2009) 3352.

17 J. C. Hu and K. A. Athanasiou: Tissue Eng. 12 (2006) 1337.

18 D. Wendt, N. Timmins, J. Malda, F. Janssen, A. Ratcliffe, G. Vunjak-Novakovic, and I. Martin: Tissue Eng., eds. C. V. Blitterswijk, P. Thomsen, A. Lindahl, J. Hubbell, D. F. Williams, R. Cancedda, J. D. D. Bruijn, and J. Sohier, (Academic Press, Burlington, 2008) p. 483.

19 P. Rolfe: Meas. Sci. Tech. 17 (2006) 578.

20 J. Seidel, M. Pei, M. Gray, R. Langer, L. Freed, and G. Vunjak-Novakovic: Biorheology 41 (2004) 445.

21 D. Bartis and J. Pongrácz: Hungary: University of Pécs (2011).

22 G. Vunjak-Novakovic, B. Obradovic, I. Martin, P. M. Bursac, R. Langer, and L. E. Freed: Biotech. Prog. 14 (1998) 193.

23 C.-H. Chang, H.-C. Liu, C.-C. Lin, C.-H. Chou, and F.-H. Lin: Biomaterials 24 (2003) 4853.

24 H.-C. Chen, H.-P. Lee, M.-L. Sung, C.-J. Liao, and Y.-C. Hu: Biotech. Prog. 20 (2004) 1802.

25 E. Tognana, F. Chen, R. Padera, H. Leddy, S. Christensen, F. Guilak, G. Vunjak-Novakovic, and L. Freed: Osteo. Cart. 13 (2005) 129.

26 S. Marlovits, B. Tichy, M. Truppe, D. Gruber, and W. Schlegel: Inter. J. Artif. Org. 26 (2003) 319.

27 S. Saini and T. M. Wick: Biotech. Prog. 19 (2003) 510.

28 C. K. Kuo, W.-J. Li, R. L. Mauck, and R. S. Tuan: Cur. Opin. Rheumat. 18 (2006) 64.

29 S. Saini and T. M. Wick: Tissue Eng. 10 (2004) 825.

30 E. M. Bueno, B. Bilgen, and G. A. Barabino: Tissue Eng. 11 (2005) 1699.

31 J.-L. Chen, L. Duan, W. Zhu, J. Xiong, and D. Wang: Stem cells 8 (2014) 14.

32 C.-H. Chang, F.-H. Lin, C.-C. Lin, C.-H. Chou, and H.-C. Liu: J. Biomed. Mat. Res., B 71 (2004) 313.

33 H.-C. Chen and Y.-C. Hu: Biotech. Letters 28 (2006) 1415.

34 D. Wendt, A. Marsano, M. Jakob, M. Heberer, and I. Martin: Biotech. Bioeng. 84 (2003) 205.

35 D. Pazzano, K. A. Mercier, J. M. Moran, S. S. Fong, D. D. DiBiasio, J. X. Rulfs, S. S. Kohles, and L. J. Bonassar: Biotech. Prog. 16 (2000) 893.

36 R. Santoro, A. L. Olivares, G. Brans, D. Wirz, C. Longinotti, D. Lacroix, I. Martin, and D. Wendt: Biomaterials 31 (2010) 8946. 
37 J. Liao, X. Guo, K. J. Grande-Allen, F. K. Kasper, and A. G. Mikos: Biomaterials 31 (2010) 8911.

38 A.-M. Freyria, Y. Yang, H. Chajra, C. Rousseau, M.-C. Ronzière, D. Herbage, and A. E. Haj: Tissue Eng. 11 (2005) 674.

39 T. Davisson, R. L. Sah, and A. Ratcliffe: Tissue Eng. 8 (2002) 807.

40 X. Xu, J. P. Urban, U. Tirlapur, M.-H. Wu, Z. Cui, and Z. Cui: Biotech. Bioeng. 93 (2006) 1103.

41 S. E. Carver and C. A. Heath: Tissue Eng. 5 (1999) 1.

42 R. W. Forsey, R. Tare, R. O. Oreffo, and J. B. Chaudhuri: Biotech. Appl. Biochem. 59 (2012) 142.

43 E. Gilbert, M. Mosher, A. Gottipati, and S. Elder: Processes 2 (2014) 658.

44 E. Wernike, Z. Li, M. Alini, and S. Grad: Cell Tissue Res. 331 (2008) 473.

45 C. Lee, A. Grodzinsky, and M. Spector: J. Biomed. Mat. Res., A 64 (2003) 560.

46 A. Kumar and B. Starly: Biofabrication 7 (2015) 044103. 